\title{
Understanding the Concept of Language of Mathematics for Effective Teaching and Learning of Mathematics in a School System
}

\author{
Joseph Ugochukwu Joseph \\ Department of Mathematics Education, University of Agriculture, Makurdi, Nigeria \\ profjoseph4@gmail.com \\ *Corresponding Author: profjoseph4@gmail.com | Phone : +2348066351822
}

\begin{abstract}
The paper deals with understanding the concept of language of mathematics for effective teaching and learning of mathematics in a School System. It as well looked at the causes of students' failure in mathematics in internal and external examinations to include lack of understanding of mathematics language. The persistent poor performance of students in internal and external examinations in mathematics has become an issue of concern to Mathematics educators, and relevant stakeholders. Findings of recent research studies in mathematics education have identified inadequate understanding of mathematics language to translate word problems into mathematical form as one of the factors responsible for poor performance. Hence, this paper examined the language of mathematics and its understanding. Specifically, the paper discussed the algebraic language; the language of basic operations; the language of set theory; the language of functions; the language of relations; the logical language and the language of differential calculus. The paper further examined the usefulness of variables; difficulties in learning mathematical language and made some recommendations among many others to include that mathematics teachers should teach the mathematical language instruction in mathematics before proceeding to the topic proper to enhance better performances of students in mathematics.
\end{abstract}

Keywords: Mathematics; Students; Interests; Language;

\section{Introduction}

Mathematics is vital to students' future and national development and its study has been ineffective in meeting the demands of national development in Nigeria. The ultimate aim of teaching mathematics at any level of education is to develop in learners the ability to recognize problem and apply the mathematical knowledge acquired to solve problems. Researchers such as Ojerinde (1999), Odogwu (2002) and Eguavon (2002) viewed Mathematics as the communication system of concepts of shape, size, quantity and order used to described diverse phenomena. Also, Onoh and Obodo (2000) defined Mathematics as a subject in which one never knows what one talks about nor knows whether what one says is true. This means that Mathematics is a language in which its truth is dependent on the meaning assigned to the words and the symbols in Mathematics. Mathematics has its own language whose terminologies are not universal to most first spoken different cultures in the world. For example, there are no equivalent words for factorization, function, equation and so on in most Nigerian languages (Osafehinti, 1993). Also, in algebra we state that $\mathrm{x}+\mathrm{y}=\mathrm{c}$; this is a symbolic representation of certain variables. In English Language, for example, this statement means that one quantity of one thing is added to another thing to a total of get c things. If one compares the two languages, there is no doubt that Mathematical language is more precise. The importance of language in the study of topics in each branch of mathematics cannot be undermined.

Despite the importance of mathematics to students and society at large, the general achievement in school mathematics has more often than not been affected by students' poor performance in public mathematics examinations over the years in Nigeria ( Iji, Agbo-Egwu \& Adikwu, 2014). The Annual Report of the Chief Examiner of the West Africa Examination Council (WAEC, 2004 and 2007) has indicated that achievement in public mathematics paper was low among the secondary school students. The WAEC Chief Examiner's Report as cited in ( Iji, Agbo-Egwu \& Adikwu, 2014) specifically advanced reasons for students' poor performance in mathematics to include:

a. Shallow knowledge of basic principles, concepts and appropriate application of laws and formulae. 
b. Confusion between similar and related topics.

c. Lack of mastery of the subject language, resulting in misinterpretation of facts. This position alludes to the fact that the concepts in mathematics should be properly explained to the learners in order to improve upon their overall achievement in mathematics. The Chief Examiner's Report is an evident that students have problems in understanding and interrelating symbols, formulae and peculiar language used in school mathematics. Also, according to the West African Examination Council Chief Examiner's Reports (2007), students attempt of fail answered questions some problems in the Senior Secondary School Mathematics Examinations because of their inability to translate the given word expression into diagrammatical form or representation. Therefore, Mathematical language must be accurately understood for proper understanding of Mathematics concepts/topics.

It is a remarkable phenomenon that children can learn to speak without ever being consciously aware of the sophisticated grammar they are using. Indeed, adults too can live a perfectly satisfactory life without ever thinking about ideas such as parts of speech, subjects, predicates or subordinate clauses. Both children and adults can easily recognise ungrammatical sentences, at least if the mistake is not too subtle, and to do this it is not necessary to be able to explain the rules that have been violated. Nevertheless, there is no doubt that one's understanding of language is hugely enhanced by a knowledge of basic grammar and this understanding is essential for anybody who wants to do more with language than use it unreflectingly as a means to a non-linguistic end (Gay, 2008). The same is true of mathematical language. Up to a point, one can do and speak mathematics without knowing how to classify the different sorts of words one is using, but many of the sentences of advanced mathematics have a complicated structure that is much easier to understand if one knows a few basic terms of mathematical grammar. Mathematics has its own language, much of which we are already familiar with. For example the digits $0,1,2,3,4,5,6,7,8,9$ are part of our everyday lives. Whether we refer to 0 as 'zero', 'nothing', 'nought', or ' $\mathrm{O}$ ' as in a telephone number, we understand its meaning. There are many symbols in mathematics and most are used as a precise form of shorthand. We need to be confident when using these symbols, and to gain that confidence we need to understand their meaning. To understand their meaning, there are two things to help us

a. Context - this is the context in which we are working, or the particular topics being studied, and

b. Convention - where mathematicians and scientists have decided that particular symbols will have particular meaning.

Mathematics, like other specialized areas of human activities, has special forms and ways of using language. The development of mathematics over the years has also led to the development of new words, new notations and conventional styles of argument and communication. Mathematical language can be viewed as shorthand and very much represented by symbols. Mathematics has its own vocabulary. Some mathematical words may not be encountered outside the mathematics classroom. Examples of such words include quadrilateral, parallelogram, hypotenuse, etc. There are also mathematical words that have been borrowed from common English language. These are face, power, product, rational, etc. Some mathematical words have either Latin or Greek roots. For example, the word "isosceles" is derived from two Greek words, iso meaning equal and skelos meaning leg. Tangent is a latin word which means to touch (Morgan, 2000). Mathematics is like a language, although technically it is not a natural or informal human language, but a formal, that is, artificially constructed language. Importantly, we use our natural everyday language to teach the formal language of mathematics. Sometimes we encounter problems when the technical words we use, as formal parts of mathematics, conflict with an everyday understanding or use of the same word, or related words (Gough, 2007). Mathematics uses many words in the English language that are already familiar to students in their everyday lives. Words such as 'change' have a specific mathematical meaning, but as they also have an everyday meaning, they are ambiguous in mathematics classrooms. Some everyday English words can create confusion because their mathematical meanings are different from their everyday meaning, e.g. root, odd, improper and irrational. Some of these words have negative connotations that can hinder the understanding of their mathematical meaning. The English meaning can also interfere with mathematical language and their meaning in other ways. Many students understand straight lines as lines drawn straight up and down or straight across the page, i.e., vertical or horizontal. This may interfere with students' understanding that the diagonal of a quadrilateral and polygons are also straight lines. Another area of difficulty with daily use of English and mathematical usage is the following words "or", "some", "all" and "any". For example, show that the sum of any two odd numbers is "even". Some pupils believe that one example suffices for the example, e.g., $3+7=10$. They may also interpret the question to mean "any" example proves the statement. In mathematics, the word "any" is used to mean a generality while in everyday English it is merely arbitrariness. Therefore, as a mathematics teacher, your evaluation should reveal the students' understanding of a word as either a mathematical proof or as used in English language. The main reason for the importance of mathematical language is that the statements of mathematics are 
supposed to be precise, and it is not possible to achieve a high level of precision unless the language one uses is free of many of the vaguenesses and ambiguities of ordinary speech. Mathematical sentences can also be highly complex: if the parts that made them up were not clear and simple, then the unclarities would rapidly propagate, multiply and render the sentences unintelligible.

Mathematical Symbolism

Mathematics uses a lot of symbols to help in the solution of many scientific, mathematical and complicated problems. Many of these can be a challenge to students' learning if they are not understood. As students progress from one level of education to another, their progress in mathematics depends on their ability to employ mathematical language and symbols correctly. The ability to do so helps them even in other subjects that use these symbols to simplify their own texts. The symbols for the four basic mathematics operations of addition, subtraction, multiplication, division and equality symbols are well known to all students at all level. Some other important symbols students commonly used in mathematics lessons are:

$>$ for greater than

$<$ for less than or

$\wedge$ for angle

// for parallel

$\equiv$ for Congruency

$\sqrt{ }$ for square root

for summation and many others

\section{The Algebraic Language}

Algebra brings with it a lot of mathematical symbols that pose difficulty for students. Morgan (2003) lists the problems students encounter in the learning of algebraic symbols as follows:

1. Reading text includes mathematical language which involves different skills from those needed for ordinary English text. Moreover, whereas the English text can be read in order from left to right, some mathematical symbols require the students to attend to them in a non-linear form, example $(3 / 5+3) 3$ or $\frac{3 x+4}{5 \times 2-3}$

2. Some students may find it difficult to cope with some mathematical symbols except they are read aloud, either to themselves or others. The introduction of greek letter like $\alpha, \theta, \gamma$ further complicate and symbol. Therefore, teachers of mathematics should not take them as extension of algebraic symbols. The teacher should rather give greater attention to helping students develop understanding and meaning of these symbols. You will find that Greek letters are used in many calculations. For example, the Greek letter 'pi', written $\Pi$, is used to represent the number 3.14159....This number continues forever without repeating. We often use $\alpha$ ('alpha'), $\beta$ ('beta'), and $\theta$ ('theta') to represent angles. The Greek capital letter 'sigma' or $\Sigma$ is frequently used to represent the addition of several numbers. The communication, written and oral, should incorporate these symbols.

3. The common use of the analogy that mathematics symbols are shorthand does not provide conceptual understanding of these symbols, e.g., "a" stands for apple and " $b$ " for banana. These may hinder pupils and students understanding mathematical symbols because it does not give the generalized meaning of these symbols.

4. The different way of "saying" or "reading" the same symbols may be a challenge to students. For example, the following expressions can be said correctly in not just one single way.

$5(x+3)$ is 5 bracket $x+3$ or 5 into $(x+3)$ bracket

$\frac{3 x-4}{2}$ is $3 x-4$ bracket over 2 or $3 x-4$ all over 2

(A U B) $U$ C is A union B all intersection C or A intersection C union B intersection C.

The positioning of numbers and symbols in relation to each other also gives meaning. For example, you will come across use of superscripts. These are small numbers or symbols written at the top right of another, as in 42 . In this context 42 is shorthand for 'four squared' or $4 \times 4$. Similarly 43 is shorthand for 'four cubed' or $4 \times 4 \times 4$. On the other hand 32o can mean different things in different contexts. It might mean an angle of 32 degrees. It could mean 32 to the power zero, which is actually 1 . In printed work characters with slightly different shapes and sizes are used - compare 32o meaning 32 degrees and 320 meaning 32 to the power 0. Clearly these have very different meanings. You need to know the context. $32{ }^{\circ} \mathrm{C}$ is a temperature of 32degrees Celsius. What about 6,3? This could mean several things. But with brackets around, $(6,3)$ can mean a pair of coordinates used to plot a point on a graph. Brackets can mean a variety of different things. For example, in the study of probability you will come across expressions like $p(H)=\frac{1}{2}$, this means the probability of scoring a Head, when tossing a coin, is $\frac{1}{2}$. Again, knowing the context is vital. The symbol $\%$ is a percentage sign and means 'out of 100 ' as in $90 \%$ meaning 'ninety out of one hundred'. $\sqrt{ }$ is a square root 
sign. For example $\sqrt{ } 16$ is the number which when multiplied by itself is 16 , that is 4 or $-4 . \bar{x}$, read as ' $x$ bar' is the mean of a set of numbers. The teacher should note that these symbols add to students anxiety in the learning of mathematics. They should, therefore, not introduce many of them in a single lesson. One way to demystify symbols is to introduce them as a way of generalizing students' findings and patterns, example, the number of squares needed to surround a square picture of side " $n$ " is what? The students' activity will lead to the general pattern of $4(n)+4$. The mathematics teacher should also collaborate with the English teachers to help in this area, by giving comprehension passages that have some mathematical connotations.

\section{The Language of Basic Operations}

Basic operations in mathematics includes: (a) + meaning plus or addition. (b) - meaning subtraction or take away. (c) $\times$ meaning multiplication or times. (d) $\div$ meaning division.

\section{a. The symbol (+)}

Words associated with this symbol are 'plus', 'add', 'increase' and 'positive'. As it stands, ' + ' clearly has some sort of meaning, but we really need to understand it within a context.

So, for example, if we see the + symbol written in the sum $2+3$ we understand that the context is one of adding the two numbers, 2 and 3, to give 5 . So here, the symbol + is an instruction to add two numbers together.

\section{b. The symbol (-)}

Words associated with this symbol are 'minus', 'subtract', 'take away', 'negative' and 'decrease'. Again, to understand the symbol we need a context. So, if we see the - symbol written in the sum $6-4$ we know this means 6 subtract 4 , and we know the answer is 2 . In a different context, we might see $-5{ }^{\circ} \mathrm{C}$, meaning a temperature of minus five degrees Celsius, that is five degrees below zero.

c. The symbol $(x)$

Words associated with this symbol are 'multiply', 'lots of', and 'times'. This is really just a shorthand for adding. For example, if we see

$6+6+6+6+6$ we have five lots of six, or five sixes, and in our shorthand we can write this as $5 \times 6$. Suppose we have $a+a+a+a+a$.We might write this expression as $5 \times a$. However, in this context, especially in hand-written work, we may confuse the $\times$ symbol with the letter $x$, and so we would often write simply 5a. We see that our shorthand has become even shorter. Multiplication is one of those rare occasions when we can omit a symbol altogether.

\section{d. The division symbol $(\div)$}

Division is symbolised in several different ways. For example $10 \div 5, \frac{10}{5}, 10 / 5$ are three equivalent ways of writing ten divided by 5 . We might also read this as 'how many times will 5 go into 10 ?'

The equality sign (=) and its variants

Another symbol used frequently is the equals sign $=$. The equality sign does not mean anything on its own we need a context. For example, in the sum $1+2=3$, what we are saying is that whatever we have on the lefthand side is exactly equal to whatever we have on the right-hand side. Variations on the equals sign are

$\neq$ which means 'is not equal to'

$\approx$ which means 'is approximately equal to'

$\geq$ which means 'is greater than or equal to', e.g. $x \geq 2$ means that $x$ can equal 2 , but it might also be any value larger than 2 .

$\leq$ means 'is less than or equal to', e.g. $y \leq 7$ means that y might equal 7 or might be any number less than 7 .

\section{The Language of Set Theory}

A set is a well-defined list or collection of objects with some characteristics which are unique to its members. A set is usually denoted by capital letters and the elements in it can be defined either by making a list of its members. Example, $A=\{2,3,5,7\}, B=\{a, b, c, d, e, f, g, h, i\}$ etc. The elements in a set can be defined also by describing the rule or property that connects its members. Example, $C=\{$ even number between 7 and 15 . $\mathrm{D}=$ \{set of numbers divisible by 5 between 1 and 52.$\}, \mathrm{B}=\{\mathrm{x}: \mathrm{x}$ is the factors of 24\}etc. A set can also be specified using the set-builder notation. Set-builder notation is an algebraic way of representing sets using a mixture of word, letters, numbers and inequality symbols e.g. $B=\{x: 6 \leq x<11, x \in z\}$ or $B=\{x / 6 \leq x<11, x$ $\in z\}$. The expression above is interpreted as " $B$ is a set of values $x$ such that 6 is less than or equal to $x$ and $x$ is less than 11, where $x$ is an integer $(z)^{\prime \prime}$. The stroke $(/)$ or colon (:) can be used interchangeably to mean "such that". The letter $\mathrm{Z}$ or I if used represents integer or whole numbers. Hence, the elements of the set $\mathrm{A}=$ $\{x: 6 \leq x<11, x \in z\}$ are $A=\{6,7,8,9,10\}$. The values of $x$ starts at 6 because $6 \leq x$. The values end at 10 because $x<11$ and 10 is the first integer less than 11. The Union of Sets A and B is the Set that is formed 
from the elements of the two Sets A and $\mathrm{B}$. This is usually denoted by " $\mathrm{AB}$ " meaning A Union $\mathrm{B}$. Thus $\mathrm{A} U \mathrm{~B}$ is the Set which consists of elements of $A$ or of $B$ or of both $A$ and $B$. The intersection of Sets $A$ and $B$ is the set of elements that are common to both $A$ and $B$. This is usually denoted by " $A \cap B$ " meaning A intersection $B$. If $A \cap B=\varnothing$, then the Sets $A$ and $B$ are said to be disjoint. Disjoint Sets are Sets that have no element in common. If $A$ is a Subset of the Universal Set $\xi$, then, the complement of the Set A are made up of elements that are not in A, but are found in the Universal Set $\xi$. This is usually denoted by Ac or A'.

\section{The Language of Functions}

In mathematics, a function is a relation between a set of inputs and a set of permissible outputs with the property that each input is related to exactly one output. A function $\mathrm{f}$ takes an input $\mathrm{x}$, and return a single output $f(x)$. An example is the function which relates each real number $x$ to its square $x 2$. In the example, if the input is -3 , then the output is 9 , and can be written as $f(-3)=9$. Likewise, if the input is 3 , then the output is also 9. Without the understanding of the language of function, it will be difficult for the learners of school mathematics to relate numbers. To specify a function, therefore, one must be careful to specify two sets as well: the domain, which is the set of objects to be transformed, and the range, which is the set of objects they are allowed to be transformed into. A function $\mathrm{f}$ from a set $\mathrm{A}$ to a set $\mathrm{B}$ is a rule that specifies, for each element $x$ of $A$, an element $y=f(x)$ of $B$. (Not every element of the range needs to be used: consider once again the example of "two times" when the domain and range are both the set of all positive integers.) The following symbolic notation is used: $f: A \rightarrow B$ means that $f$ is a function with domain $A$ and range $B ; f(x)=y$ means that $\mathrm{f}$ transforms $\mathrm{x}$ (which must be an element of $\mathrm{A}$ ) into $\mathrm{y}$ (which must be an element of $\mathrm{B}$ ); $\mathrm{f}: \mathrm{x} \rightarrow \mathrm{y}$ is another way of writing $f(x)=y$ that is sometimes more convenient.

\section{The Language of Relations}

A relation between two sets is a collection or ordered pairs containing one object from each set. If the object $x$ is from the first set and the object $y$ is from the second set, then the object are said to be related if the ordered pair $(x, y)$ is in the relation. Relation is used to describe certain properties of things. It is clear, that things are either related, or they are not, there are no in-betweens. Formally, a relation is a set of n-tuples of equal degree. Thus a binary relation is a set of pairs, a ternary relation is a set triples, and so forth. In the language of set theory, a relation between two sets is a subset of their Cartesian product. The use of the term "relation" is often used as shorthand to refer to binary relations, where the set of all the starting points is called the domain and the set of the ending points is the range. The domain is the $x^{\prime}$ s and the range is the $y^{\prime} s$.

\section{The Logical language and Statements}

Mathematical logic can be defined as the study of the relationship between certain objects such as numbers, functions, geometric figures etc. Statements are verbal or written declarations or assertions. The fundamental (i.e logical) property of a statement is that it is either true or false but not both. So logical statements are statements that are either reasonably true or false but not both. To determine the truth or falsity of a simple statement, one requires pre-knowledge and/or definition of the main concepts related to the statements. For example, the simple statement 'it is hot' is true if 'it' refers to a hot object or weather. Otherwise the statement is false. A true statement is said to have a truth value $T$ while a false statement is said to have a truth value $F$. The opposite of a statement is called the negation of the statement. Given any logical statement $\mathrm{P}$, the negation (or the contradiction or the denial) of $\mathrm{P}$ is written symbolically as $\sim \mathrm{P}$. When two or more simple statements are combined, we have a compound statement. To do this, we use the words: 'and', 'or', 'if ... then', 'if and only if', 'but'. Such words are called connectives. Conjunction (or $\wedge$ ) of logical reasoning: Any two simple statements $\mathrm{p}, \mathrm{q}$ can be combined by the word 'and' to form a compound (or composite) statement ' $\mathrm{p}$ and $\mathrm{q}$ ' called the conjunction of $\mathrm{p}, \mathrm{q}$ denoted symbolically as $\mathrm{p} \wedge \mathrm{q}$. Any compound statement formed by using the word 'or' to combine simple statements is called a disjunction. The symbol ' $v$ ' stands for 'or'. When the connective 'if...then' is used to combine simple statements, the result is called an implicative or conditional proposition. We denote implication symbolically by $\Rightarrow$ i.e $p \Rightarrow q$ means if $p$ is true, then $\mathrm{q}$ is true. (or $\mathrm{p}$ implies q or $\mathrm{p}$ only if $\mathrm{q}$, etc.) Another common statement in Mathematics is of the form " $p$ if and only if $q$ ". This statement is actually the combination of two conditional statements and so it is called bi-conditional or equivalence and is denoted by $\mathrm{p} \leftrightarrow \mathrm{q}$ or sometimes $\mathrm{p}$ iff $\mathrm{q}$ (if and only if) i.e implies and is implied by.

\section{List of logical operators and symbols}

The word 'not' and the four connectives 'and', 'or', 'if ... then', 'if and only if' are called logic operators. They are also referred to as logical constants. The symbols adopted for the logic operators are given below. 


\begin{tabular}{cc}
\hline Logic Operators & Symbols \\
\hline 'not' & - or $\sim$ \\
\hline 'and' & $\wedge$ \\
\hline 'or' & v \\
\hline 'if ... then' & $\rightarrow$ \\
\hline 'if and only if' & $\leftrightarrow$ \\
\hline
\end{tabular}

When the symbols above are applied to propositions $\mathrm{p}$ and $\mathrm{q}$, we obtain the representations in the table below:

\begin{tabular}{ll}
\hline Logic operation & Representation \\
\hline 'not $\mathrm{p}^{\prime}$ & $\sim \mathrm{p}$ or $\overline{\mathrm{p}}$ \\
\hline 'P and $\mathrm{q}^{\prime}$ & $\mathrm{p} \wedge \mathrm{q}$ \\
\hline ' $\mathrm{p}$ or $\mathrm{q}$ & $\mathrm{p} \vee \mathrm{q}$ \\
\hline
\end{tabular}

It behoves the teacher to give attention to helping students develop understanding and meaning of these symbols.

\section{The Language of Differential Calculus}

Suppose that $\mathrm{x}$ and $\mathrm{y}$ is real numbers and that $\mathrm{y}$ is a function of $\mathrm{x}$, that is, for every value of $\mathrm{x}$, there is a corresponding value of $y$. This relationship can be written as $y=f(x)$, if $f(x)$ is the equation for a straight line (called a linear equation), then there are two real numbers $m$ and $b$ such that $y=m x+b$ in this 'slope intercept form', this term $\mathrm{m}$ is called slope and can be determined from the formula.

$\frac{\text { change in } \mathrm{y}}{\text { change in } \mathrm{x}}=\frac{\Delta \mathrm{y}}{\Delta \mathrm{x}}$

Where the symbol $\Delta$ (the uppercase form the Greek letter Delta) is an abbreviation for "change in". It follows that $\Delta y=m \Delta x$. A general function is not a line so it does not have a slope. Geometrically, the derivative of $\mathrm{f}$ at the point $\mathrm{x}=\mathrm{a}$ is the slope of the tangent line to the function at the point $\mathrm{a}$. This is often denoted in language notation or $\frac{d y}{d x} l x=a$ in Leibniz notation. Since the derivative is the slope of the linear approximation to $f$ at the point $a$, the derivative (together with the value of $f$ at a) determines the best linear approximation, or linearization, of $f$ near the point a. If every point $a$ in the domain $f$ has a derivative, there is a function that send every point a to the derivative of $f$ at a. for example, if $f(x)=x 2$, then the derivative function $\mathrm{fl}(\mathrm{a})=\frac{\mathrm{dy}}{\mathrm{dx}}=2 \mathrm{x}$. A closely related notion is the differential of a function. When $\mathrm{x}$ and $\mathrm{y}$ are real variables, the derivative of $f$ at $x$ is the slope of the tangent line to the graph $f$ at $x$. because the source and target of $\mathrm{f}$ are one dimensional, the derivative of $\mathrm{f}$ is the real number. If $\mathrm{x}$ and $\mathrm{y}$ are vector, then the best linear approximation to the graph of $\mathrm{f}$ depends on how $\mathrm{f}$ changes in several irections at once. Taking the best linear approximation in a single direction determines a partial derivative, which is usually denoted by $\frac{\partial y}{\partial x}$. The linearization of $\mathrm{f}$ in all directions at once is called total derivative.

The usefulness of Variables in School Mathematics.

Variables are another form of mathematical symbol. These are used when quantities take different values. Imagine taking a car journey and think about the speed at which you are travelling. As you travel along your speed may change. So, speed is a variable - that is, a quantity which can change. We will be using letters to stand for quantities like this. For example, we might use the letter $v$ for speed. To a large extent we can use any letter we choose, although there are conventions. We might choose to use $\mathrm{d}$ for distance and $\mathrm{t}$ for time (Gay, 2008). By convention, we use $\mathrm{u}$ to be an initial speed, and $\mathrm{v}$ to be a final speed. In a different context, $\mathrm{v}$ might refer to volume. We need to check the context to fully understand the meaning. If we see $\mathrm{v}=\mathrm{d} / \mathrm{t}$ where $\mathrm{d}=$ distance, and $\mathrm{t}=$ time, then we would know that $\mathrm{v}$ is a speed. On the other hand, if we see $\mathrm{v}$ $=\frac{4}{3} \Pi \mathrm{r} 3$ where $\mathrm{r}$ is the radius of a sphere, we know that $\mathrm{v}$ stands for the volume of the sphere.

\section{Difficulties in Learning Mathematical Language}

Researchers (Gay, 2008; Rubenstein \& Thompson, 2002; Rubenstein, 2007; Thompson \& Rubenstein, 2000) reveal numerous categories of difficulty for students' misunderstandings of mathematical vocabulary, thus hindering their overall mathematical communication, learning, and achievement which is why is direct instruction of vocabulary is deemed necessary:

1. Some words are shared by mathematics and everyday English and have comparable meanings, but they have a more distinct meaning in mathematics.

When students hear these words used across outside of the context of mathematics and then inside the mathematics classroom, they often struggle to decode how the terms in their everyday language is 
distinctly different from the mathematical definition. A few examples of terms that fit this category are: even, odd, power, right, similar, event, factor, prime, expression, base, radical, combination, function, mode, limit, slope, difference, reflection, variable, and foot.

2. Some mathematical terms are homonyms or homophones with everyday English words.

Aligning with the first category of difficulty, words like some, pie and compliment are possible every day usage vocabulary words for students. When introduced to these words in a mathematical context, they may misunderstand or misuse the word based on a student's prior knowledge. Explicitly addressing these misconceptions may help enhance students' conceptual understanding.

3. Some words have more than one mathematical meaning when used as a different part of speech. For instance when defining a four sided figure with all congruent sides and four right angles, it is defined as a square. Yet, when one squares a number, he/she multiplies the number by itself to get a perfect square. Also these two concepts can be bridged pictorially and conceptually, effective vocabulary instruction on usage of the term in context may clarify misconceptions for students. Other examples are: round, range, base, second, and side.

4. Some words are found only in mathematical contexts.

Some of these terms include: quotient, denominator, isosceles, polynomial, hypotenuse, asymptote, hyperbola, algorithm, integer, parallelogram, and quadrilateral.

5. Some mathematical concepts are verbalized in more than one way.

One example of this is the fraction one-fourth. When talking in terms of money or football, one-fourth may be communicated as a quarter. Although "as adults, we are comfortable with the varied meanings and implied understandings in words and phrases" (Rubenstein \& Thompson, 2002, pg. 107), students are not. For conceptual purposes, we need to address these vocabulary issues for students to be able to have a deeper understanding and be able to better communicate mathematically.

6. Some words are learned in pairs that often confuse students.

A few examples of these include: multiple and factor, radius and diameter, area and perimeter, numerator and denominator, and hundreds and hundredths. Although often words taught together are related mathematically, focusing on two concepts together without clear definitions distinguishing differences, students may mix up the two words and lack the conceptual understanding to apply or communicate their understandings.

7. Some mathematical words shared with other disciplines have different technical meanings in the two contexts.

Some words in mathematics are also explicitly taught in science, English/Language Arts, or Social Studies. If educators do not clarify a term's specific mathematical definition, students may assume the term is the same across content areas, which would result in a misunderstanding. A few examples to fit this category of difficulty are: prism, median, power, degree, divide, and variable.

8. Modifiers change meanings of words in critical ways.

A few examples of these include: bisector versus perpendicular bisector, equation versus linear, quadratic, and cubic equation, and trapezoid versus right trapezoid. These modifiers (adjectives) describing the noun may seem clear cut, but for some students, putting the terms together may cause some discrepancies.

9. Students may adopt an informal terms as if it is mathematical.

This would cause a divergence in mathematical discourse, which would cause for an excellent classroom discussion, but with being brought to attention, students may misalign mathematical terms with similar terms. Two examples of this would include diamond versus a rhombus and a corner versus a vertex.

Being knowledgeable about how these particular challenges for students' comprehension in understanding vocabulary terminology may be a teacher's first step in enhancing students' overall math learning and achievement in the classroom. In addition to understanding these difficulties, a teacher must research best practices to help students overcome these challenges. Throughout the literature, many researchers propose how to bridge these conceptual gaps through the explicit teaching of mathematic vocabulary.

\section{Conclusion}

The paper examined the Language of Mathematics and its understanding. This is in accordance to the WAEC Chief Examiner's Report as cited in (Iji, Agbo-Egwu \& Adikwu, 2014), stated that shallow knowledge 
of basic principles, concepts and appropriate application of laws and formulae; confusion between similar and related topics; and lack of mastery of the subject language resulting in misinterpretation of facts. The WAEC Chief Examiner's Report (2007) is an evident that students have problems in understanding and interrelating symbols, formulae and peculiar language used in school mathematics.

Mathematics educators all levels should endeavour that learners of mathematics have proper understanding of The Language of Mathematics. Basic concepts in mathematics should be properly explained to the learners in order to improve upon their overall achievement in mathematics. ability to translate a given word expression into diagrammatical form or representation will foster better performance of students in school mathematics Therefore, Mathematical language must be accurately understood for proper understanding of Mathematics concepts/topics.

\section{Recommendations}

The following recommendations are considered appropriate:

1. Mathematics teachers should teach the Mathematical language instruction in mathematics before proceeding to the topic proper to enhance better performances of students in mathematics.

2. Mathematics teachers in public schools should pay more attention to initial giving the Mathematical language instructions in mathematics to enhance students' performance in solving word problems.

3. The content of word problems in general should be related to real life situation of practical events in the environment capable of supervising students intellect endurance.

4. There should be regular training and retraining of teachers through Seminars and Workshops to improve their skills and methodologies in handling word problems in Mathematics.

\section{References}

Ameen S.K (2007). Effect of Mathematical Language instruction on Senior Secondary School Students' Performance in Bearing word problems in Mathematics. Unpublished M.ED. dissertation, University of Illorin, Illorin.

Eguavon, O.J. (2002). Mathematics the Heart of Information Technology. Implication for Secondary Education. Preceedings of the Annual National Conference of the Mathematical Association of Nigeria (September $2^{\text {nd }}-6^{\text {th }}$ )

Gay, S. (2008). Helping teachers connect vocabulary to conceptual understanding. The Mathematics Teacher, 102(3), 218-223.

Gough, J. (2007). Conceptual complexity and apparent contradictions in mathematics language. Australian Mathematics Teacher, 63(2), 8-16. Halliday, M. A. K. (1978). Language as social semiotic. London: Edward Arnold.

Iji, C. O., Agbo-Egwu A. O., Adikwu, O(2014). Effect of metalinguistic approach on student's achievement in secondary schools statistics in makurdi metropolis, benue state. Journal of education and practice 5(9).

Morgan, A.(2003): What is narrative therapy? An easy - to - read introduction. Duiwich centre publication.

National Business And Technical Education Board, Chief Examiners' Reports NOV/DEC 2012

Odogwu, H.N. (2002). The Mathematics teacher factor in the achievement of the goals of the Universal Basic Education (UBE). Abacus: The Journal of the Mathematics Association of Nigeria, 27 (1), 72-79.

Onboh. D.O. and Obodo, G.C. (2000). The Application of Mathematical language in Technology proceeding for Annual Conference of the Mathematical Association of Nigeria.(September $1^{\text {st }} 5^{\text {th }}$ )

Ojerinde, D. (1999). Mathematics in Technology Developmen. Focus on the next Millenium Implication for Secondary Education In Nigeria. A lead paper at the $36^{\text {th }}$ Annual Conference of Mathematical Association of Nigeria. $31^{\text {st }}$ August-Sept $4^{\text {th }}$

Osefehinti, I.O. (1993). Mathematical Language and Mathematic Achievement are pre-universal levels. ABACUS: The journal of the Mathematical Association of Nigeria 23 (1). 73-87.

Rubenstein, R. (1996). Strategies to support the learning of the language of mathematics. In P. Elliott \& M. Kenney (Eds.), Communication in Mathematics, K-12 and Beyond (pp. 214-218). Reston, Virginia: National Council of Teachers of Mathematics.

Rubenstein, R. (2007). Focused strategies for middle-grades mathematics vocabulary development. Mathematics Teaching in the Middle School, 13(4), 200-207.

Rubenstein, R., \& Thompson, D. (2002). Understanding and supporting children's mathematical vocabulary development. Teaching Children Mathematics , 9(2), 107-112.

Salman, M.F. (2002). The National Policy on Education and its Implication for Mathematics Education in the Universal Basic Education. ABACUS: The Journal of the Mathematical Association of Nigeria 27 